\title{
Avaliação da condição bucal de pacientes em ventilação mecânica internados em Unidade de Terapia Intensiva
}

\author{
Assessment of the oral condition of patients on mechanical ventilation admitted to the Intensive \\ Care Unit \\ Evaluación de la condición bucal de los pacientes en ventilación mecánica ingresados en la Unidad \\ de Cuidados Intensivos
}

Recebido: 23/04/2021 | Revisado: 03/05/2021 | Aceito: 04/05/2021 | Publicado: 18/05/2021

Yasmin Alves do Nascimento

ORCID: https://orcid.org/0000-0003-4840-7830

Universidade Federal de Sergipe, Brasil

E-mail: amin.odonto@gmail.com

Graziane Ribeiro Couto

ORCID: https://orcid.org/0000-0002-4443-7867

Universidade Federal de Sergipe, Brasil

E-mail: grazianeribeiro.couto@gmail.com

Helga Lucy Santos Feitosa de Melo

ORCID: https://orcid.org/0000-0002-1254-9477

Fundação de Beneficência Hospital de Cirurgia, Brasil

E-mail: helgafeitosa@hotmail.com

Mônica Christine Alves Cabral Cardoso

ORCID: https://orcid.org/0000-0001-9030-4726

Universidade Federal de Sergipe, Brasil

E-mail: monica_chriscabral@ hotmail.com

José Augusto Santos da Silva

ORCID: https://orcid.org/0000-0003-1515-5585

Fundação de Beneficência Hospital de Cirurgia, Brasil E-mail: ajaugust@outlook.com.br

Flaviani Alves Santana Alfano

ORCID: https://orcid.org/0000-0003-0919-2144

Fundação de Beneficência Hospital de Cirurgia, Brasil E-mail: flavi_alfano@hotmail.com

\begin{abstract}
Resumo
O objetivo do presente estudo foi avaliar clinicamente a condição odontológica de pacientes internados em uma Unidade de Terapia Intensiva (UTI). Trata-se de um estudo exploratório, descritivo e transversal com avaliação odontológica de 40 pacientes internados na UTI Geral da Fundação de Beneficência Hospital Cirurgia sediada em Aracaju-SE. Foram coletados os dados dos prontuários médicos relativos à idade, sexo, hábitos deletérios e fator causal de internação. A análise da condição bucal foi qualificada em três categorias: boa, regular e ruim. Foram encontrados que $67,50 \%$ dos pacientes foram do sexo feminino. Em relação à condição médica dos indivíduos, observou-se que o fator mais comum foi o respiratório, atingindo $32,50 \%$ deles, seguido pela sepse e fator neurológico, que correspondem a 17,50\% e 15,00\% dos pacientes, respectivamente. Quanto à distribuição das condições odontológicas, observaram-se que 65,00\% apresentaram condição regular, já 27,50\% dos pacientes apresentaram classificação boa e 7,50\% condição ruim. Em relação ao índice de saburra lingual segundo a área, viu-se que apenas $5,00 \%$ e $17,50 \%$ dos pacientes não apresentaram saburra, enquanto $22,50 \%$ apresentaram até $1 / 3$ da língua, $32,50 \%$ até $2 / 3$ da língua e $22,50 \%$ mais de $2 / 3$ da língua. Já em relação ao índice de saburra lingual segundo a espessura, $20 \%$ dos indivíduos possuíam saburra fina, ao passo que 47,50\% e 32,50\% apresentaram-se com papilas linguais visíveis e não visibilidade, respectivamente. Os resultados revelam que a maioria dos pacientes internados na UTI apresentava condição odontológica regular, e os focos orais mais prevalentes foram os abscessos dento alveolares e a candidíase oral.
\end{abstract}

Palavras-chave: Unidade de terapia intensiva; Patologia; Respiração artificial; Cavidade bucal; Biofilme.

\footnotetext{
Abstract

The aim of the present study was to clinically assess the dental condition of patients admitted to an Intensive Care Unit (ICU). This is an exploratory, descriptive and cross-sectional study with dental evaluation of 40 patients admitted to the General ICU of the Fundação de Beneficência Hospital Cirurgia based in Aracaju-SE. Data were
} 
collected from medical records regarding age, sex, deleterious habits and the causal factor of hospitalization. The analysis of the oral condition was classified in three categories: good, regular and bad. It was found that $67.50 \%$ of the patients were female. Regarding the medical condition of the individuals, it was observed that the most common factor was respiratory, reaching $32.50 \%$ of them, followed by sepsis and neurological factor, which correspond to $17.50 \%$ and $15.00 \%$ of patients, respectively. As for the distribution of dental conditions, it was observed that $65.00 \%$ had a regular condition, $27.50 \%$ of the patients had a good classification and $7.50 \%$ had a poor condition. Regarding the lingual coating index according to the area, it was seen that only $5.00 \%$ and $17.50 \%$ of the patients did not have coating, while $22.50 \%$ had up to $1 / 3$ of the tongue, $32.50 \%$ up to $2 / 3$ of the language and $22.50 \%$ more than $2 / 3$ of the language. In relation to the index of lingual coating according to thickness, $20 \%$ of individuals had thin coating, while $47.50 \%$ and $32.50 \%$ presented with visible and non-visible lingual papillae, respectively. The results reveal that the majority of patients admitted to the ICU had a regular dental condition, and the most prevalent oral foci were toothless abscesses and oral candidiasis.

Keywords: Intensive care unit; Pathology; Respiration artificial; Mouth; Dental plaque.

\section{Resumen}

El objetivo del presente estudio fue evaluar clínicamente la condición dental de los pacientes ingresados en una Unidad de Cuidados Intensivos (UCI). Se trata de un estudio exploratorio, descriptivo y transversal con evaluación odontológica de 40 pacientes ingresados en la UCI General del Hospital Cirurgia de la Fundación de Beneficencia con sede en Aracaju-SE. Se recogieron datos de historias clínicas sobre edad, sexo, hábitos deletéreos y factor causal de la hospitalización. El análisis de la condición bucal se clasificó en tres categorías: bueno, regular y malo. Se encontró que el 67,50\% de los pacientes eran mujeres. En cuanto a la condición médica de los individuos, se observó que el factor más común fue el respiratorio, alcanzando el 32,50\% de ellos, seguido de la sepsis y el factor neurológico, que corresponden al $17,50 \%$ y $15,00 \%$ de los pacientes, respectivamente. En cuanto a la distribución de las condiciones dentales, se observó que el $65,00 \%$ tenía una condición regular, el 27,50\% de los pacientes tenía una buena clasificación y el 7,50\% una mala condición. En cuanto al índice de recubrimiento lingual según la zona, se observó que solo el 5,00\% y el 17,50\% de los pacientes no tenían recubrimiento, mientras que el 22,50\% presentaban hasta $1 / 3$ de la lengua, $32,50 \%$ hasta $2 / 3$ de la lengua. idioma y 22,50\% más de $2 / 3$ del idioma. En relación al índice de recubrimiento lingual según espesor, el $20 \%$ de los individuos presentaba recubrimiento fino, mientras que el 47,50\% y el $32,50 \%$ presentaban papilas linguales visibles y no visibles, respectivamente. Los resultados revelan que la mayoría de los pacientes ingresados en la UCI tenían una condición dental regular, y los focos bucales más prevalentes fueron los abscesos desdentados y la candidiasis bucal.

Palabras clave: Unidad de cuidado intensivo; Patología; Respiración artificial; Cavidad bucal; Biofilm.

\section{Introdução}

A Odontologia Hospitalar é uma especialidade odontológica que objetiva a realização de cuidados e procedimentos bucais em âmbito hospitalar. Com foco no cuidado de pacientes críticos que necessitam de tratamentos meticulosos, visa a prevenção de complicações sistêmicas através da higiene bucal (HB) e tratamento das alterações bucais com o intuito de eliminar focos de infecção, remissão da dor e promoção de conforto do paciente crítico (Saldanha, Costa, Pinto, \& GaettiJardim, 2015). O paciente que adentra em uma Unidade de Terapia Intensiva (UTI) geralmente se encontra em condições de saúde gravíssimas. E devido à severidade do seu estado, quase sempre não possui autonomia em atividades cotidianas como a higiene pessoal, alimentação e excreção (Cruz, Morais, \& Trevisani, 2014). Normalmente apresentam condições de deficiência de higiene bucal, pois frequentemente permanecem com a boca aberta devido à intubação orotraqueal. Isso promove a desidratação da mucosa, com a diminuição do fluxo salivar, permitindo maior colonização de bactérias e levando a maior predisposição de doenças periodontais e focos infecciosos (Araújo, 2015).

A cavidade bucal está em contínua colonização de micro-organismos. De todas as partes do corpo humano a cavidade bucal é a que apresenta maior variedade e níveis de micro-organismos (Baeder et al., 2012). As doenças infecciosas em cavidade bucal têm sido frequentemente associadas as alterações na resposta imunológica, falta de higiene oral, desnutrição severa, tabagismo, alcoolismo e diabetes, os quais podem predispor o indivíduo ao desenvolvimento de gengivite e periodontite (Gaetti-Jardim, Setti, Cheade, \& Mendonça, 2013). As características anátomo-fisiológicas bucais são responsáveis por esta diversidade em função dos diferentes tipos de estruturas e tecidos, de acordo com a quantidade de oxigênio, a temperatura, a exposição aos fatores imunes e a disponibilidade de nutrientes (Schlesener, Rosa, \& Raupp, 2012). 
São várias espécies de bactérias, fungos e vírus residindo em um ecossistema denominado biofilme, encontrado em praticamente todos os nichos da cavidade bucal, sendo o dorso da língua e as superfícies dos dentes suas principais localizações (Morais et al., 2006). Além disso, frequentemente, em ambiente de UTI o paciente necessita de suporte de respiração artificial, demonstrando pneumonias associadas à ventilação mecânica acometendo grandes percentagens de pacientes com taxas alarmantes de mortalidade (Baeder et al., 2012).

A partir dos anos 1970, após a identificação de bactérias do biofilme dental na cavidade orofaríngea, surgiram grandes evidências de que a pneumonia aspirativa associada à ventilação mecânica poderia estar associada à composição bacteriana do biofilme dental, podendo essa colonização ser favorecida ou agravada com a higienização bucal negligenciada durante o período de internamento dos pacientes (Gaetti-Jardim, et al., 2013).

A evolução da odontologia traz subsídios significativos que comprovem a contribuição do tratamento odontológico, na prevenção e/ou melhora da condição sistêmica do paciente crítico (Araújo, 2015). A higiene bucal deficiente não supervisionada por profissionais da odontologia e focos infecciosos como doença periodontal, raízes residuais, abscessos alveolares, comuns em pacientes internados em UTI, propicia a colonização do biofilme bucal por microrganismos patogênicos, especialmente por patógenos respiratórios (Oliveira et al., 2011).

A higiene bucal satisfatória deve ser realizada primariamente a avaliação e a adequação do meio bucal. Entretanto, procedimentos odontológicos configuram procedimentos de baixa complexidade diante dos numerosos problemas apresentados pelo paciente hospitalizado, perdendo prioridade. A literatura demonstra a influência da condição bucal na evolução do quadro dos pacientes internados. A importância dos cuidados bucais, em indivíduos sob cuidados em unidades de terapia intensiva, tem sido alvo de inúmeras investigações, cujos resultados alertam para a necessidade de se implementar diretrizes para a higiene bucal destes pacientes (Ferraz et al., 2014).

De acordo com o Manual de Odontologia Hospitalar (São Paulo, 2012), uma condição bucal desfavorável interfere no prognóstico de pacientes com comprometimentos sistêmicos, onde as infecções bucais possam contribuir para a instalação de condição inflamatória sistêmica SEPSE e serve como fonte de disseminação de microrganismo por via hematogênica. A avaliação completa e a adequação do meio bucal pelo cirurgião dentista são fundamentais para minimizar e prevenir tais complicações (Saldanha et al., 2015).

Sendo assim, o objetivo deste estudo foi através de um estudo exploratório, descritivo e transversal avaliar clinicamente a condição odontológica em pacientes internados em Unidade de Terapia Intensiva Geral da Fundação de Beneficência Hospital Cirurgia sediada em Aracaju, capital do estado de Sergipe.

\section{Metodologia}

Tratou-se de um estudo de cunho transversal, desenvolvido em um período de 04 meses. Após aprovação pelo Comitê de Ética em Pesquisa em Seres Humanos da Universidade Tiradentes (911875), foi realizado um estudo com pacientes internados na UTI da Fundação de Beneficência Hospital Cirurgia (FBHC), em Aracaju/SE. Este estudo é considerado transversal porque possibilita fazer comparação entre os dados analisados (Estrela, 2018).

A população do estudo ou seus responsáveis foram orientados a respeito da pesquisa e confirmaram sua participação por meio da assinatura do Termo de Consentimento Livre e Esclarecido (TCLE) em duas vias.

A coleta de dados foi extraída dos prontuários médicos relativos à idade, gênero, fator causal de internação, hábitos deletérios e comorbidades relacionadas a problemas pulmonares. Com análise descritiva dos dados referente aos 40 pacientes, seguindo os critérios de inclusão e exclusão. Essa análise caracteriza-se por descrever a distribuição dos dados referentes à saúde levando em consideração a análise das características que se relacionam com os fenômenos estudados (Pereira, Shitsuka, 
Parreira, \& Shitsuka, 2018). Os critérios de inclusão utilizados foram: pacientes internados na UTI geral adulto do FBHC, sem capacidade de autocuidado, sob ventilação mecânica com tubo oro traqueal ou traqueostomia e com entrada na unidade durante o período da pesquisa. Os critérios para não inclusão foram: pacientes menores de idade, em ventilação espontânea, e os que se recusaram a participar da pesquisa. E para os critérios de exclusão os pacientes que possuíam deficiência neurológica que impossibilitasse o exame.

Nos voluntários foram aplicados dois métodos de avaliação da condição bucal do paciente crítico, proposto por Baeder, e o Índice de Saburra Lingual quanto à área e a espessura, proposto por Silness e Loe. As avaliações foram realizadas sob luz artificial, por um único avaliador, com experiência em UTIs, utilizando os equipamentos de proteção individual preconizados, espátula de madeira, gaze e odontoscópio. Todos os pacientes participantes do estudo, após a avaliação, receberam higiene bucal criteriosa, com gluconato de clorexidina $0,12 \%$. Os dados desta pesquisa foram coletados no mesmo momento de higiene, de modo que todos os indivíduos participantes da pesquisa fossem submetidos à avaliação com as mesmas condições de higiene bucal.

A análise da condição bucal foi baseada no Índice de Higiene Oral Simplificado, Índice Gengival e presença de placas esbranquiçadas. As condições foram reunidas em três categorias:

○ Boa, relacionada a uma condição aceitável de higiene sem sangramento ao toque ou focos de infecção aparentes;

- Regular, para aqueles pacientes que apresentavam pouco sangramento ao toque gengival e/ou candidíase observada clinicamente como placas esbranquiçadas sobre mucosa inalterada ou avermelhada, removidas mecanicamente;

○ Ruim, para pacientes com presença de abscessos e candidíase disseminada incluindo orofaringe.

Foram também registradas as presenças de lesões traumáticas, raízes residuais e abscessos dento alveolares.

A análise de saburra lingual foi baseada no Índice de saburra lingual de Silness e Loe, classificado:

- Saburra lingual quanto à área: sem saburra, saburra subclínica, saburra em até um terço da língua, em até dois terços da língua e em mais de dois terços.

- Saburra lingual quanto à espessura: saburra fina, com papilas linguais visíveis e saburra espessa, com papilas linguais sem visibilidade.

Para análise dos dados foi empregada a estatística descritiva e para testar a associação entre as variáveis foi empregado o teste exato de Fisher, uma alternativa ao teste qui-quadrado, ao nível de significância fixada em 5\% (valor p de $0,8353)$.

\section{Resultados}

Observa-se na Figura 1, a seguir, que mais de dois terços dos pacientes participantes da pesquisa são do sexo feminino $(67,50 \%)$ e mais da metade possui entre 60 e 79 anos de idade. 
Figura 1: Boxplot da idade dos pacientes participantes da pesquisa, de acordo com o sexo.

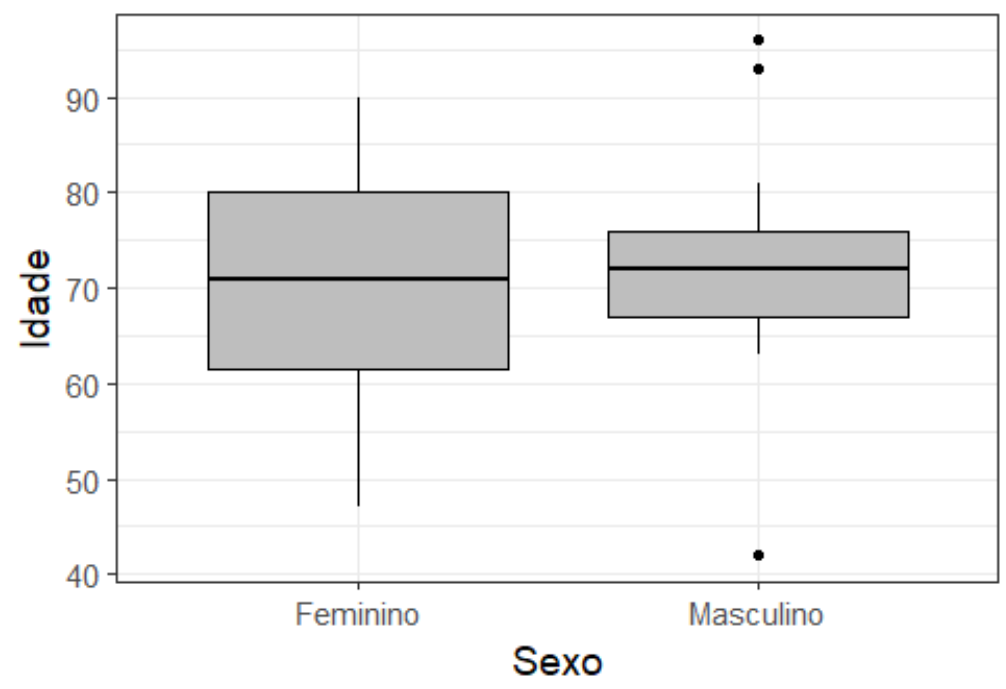

Fonte: Autores.

Em relação à condição médica dos indivíduos, vê-se que o fator de internamento mais comum foi a pneumonia, atingindo 32,50\% deles, seguido da sepse e fator neurológico, que correspondem a 17,50\% e 15,00\% dos pacientes, respectivamente. E nota- se que, apenas as condições médicas relacionadas aos fatores cardíacos e renais são mais frequentes entre os homens do que entre as mulheres. Já os portadores de diabetes mellitus atingem o mesmo número em ambos os sexos, ao passo que todas as demais condições médicas, isto é, fatores neurológicos, respiratórios, hepáticos e septicemia, atingem um número maior em mulheres do que em homens, como demonstrado abaixo na Figura 2.

Figura 2: Gráfico da frequência da condição de saúde dos pacientes participantes da pesquisa, de acordo com o sexo.

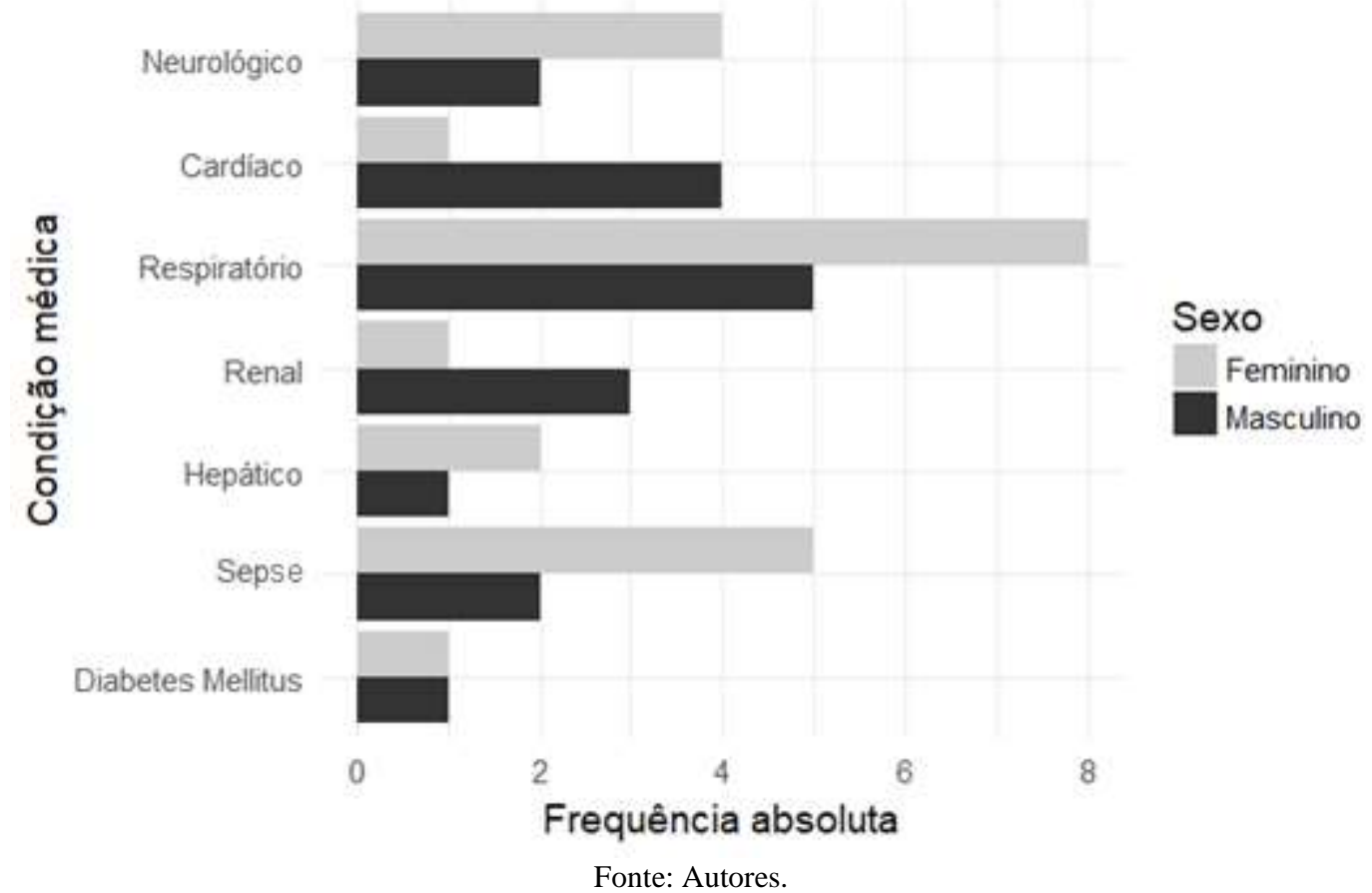

Quanto a condição odontológica, 27,50\% dos pacientes apresentaram classificação boa, 65,00\% apresentaram condição regular e 7,50\% condição ruim. E que a grande maioria dos participantes da pesquisa receberam ventilação por meio 
de tubo orotraqueal (82,50\%), enquanto que apenas 17,50\% receberam por traqueóstomo, conforme detalhado na Figura 3. Independentemente da condição odontológica dos pacientes, o tipo de ventilação mais frequente foi por tubo orotraqueal, e todos os pacientes caracterizados com condição odontológica ruim estavam sobre este tipo de tipo de ventilação.

Figura 3: Gráfico da frequência da condição odontológica dos pacientes participantes da pesquisa, de acordo com o tipo de

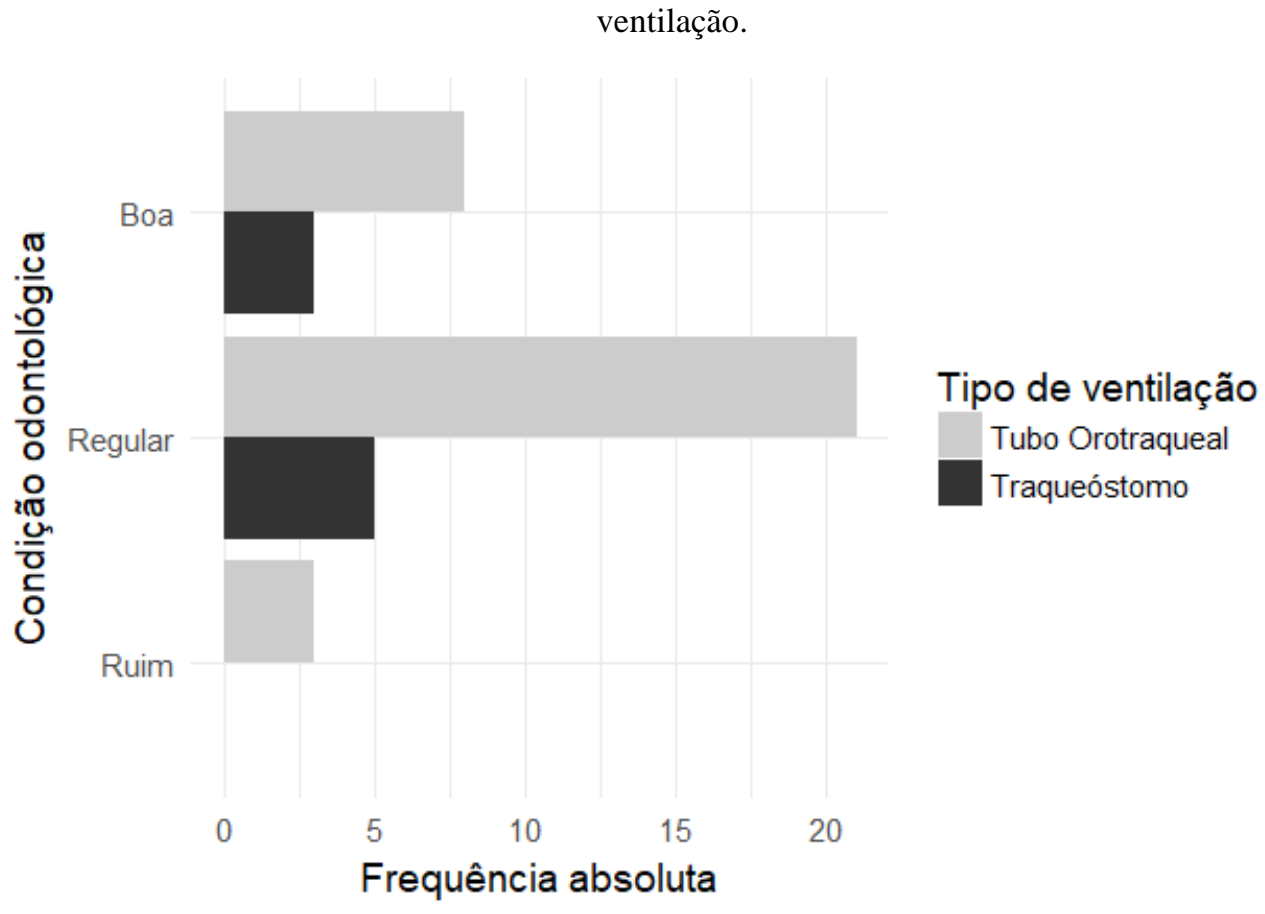

Fonte: Autores.

Ainda relacionando a condição odontológica com os hábitos dos pacientes, nota-se na Figura 4 que a grande maioria dos pacientes com condição odontológica boa não possui hábitos deletérios. E que aqueles com condição odontológica regular correspondem a metade, entretanto, alguns são etilistas, tabagistas ou apresentam ambos os hábitos. Por fim, todos os pacientes classificados com condição odontológica ruim possuem ambos os hábitos, tabagismo e etilismo, evidenciando que a condição odontológica e os hábitos estão significativamente associados. 
Figura 4: Gráfico da frequência da condição odontológica dos pacientes participantes da pesquisa, de acordo com os hábitos.

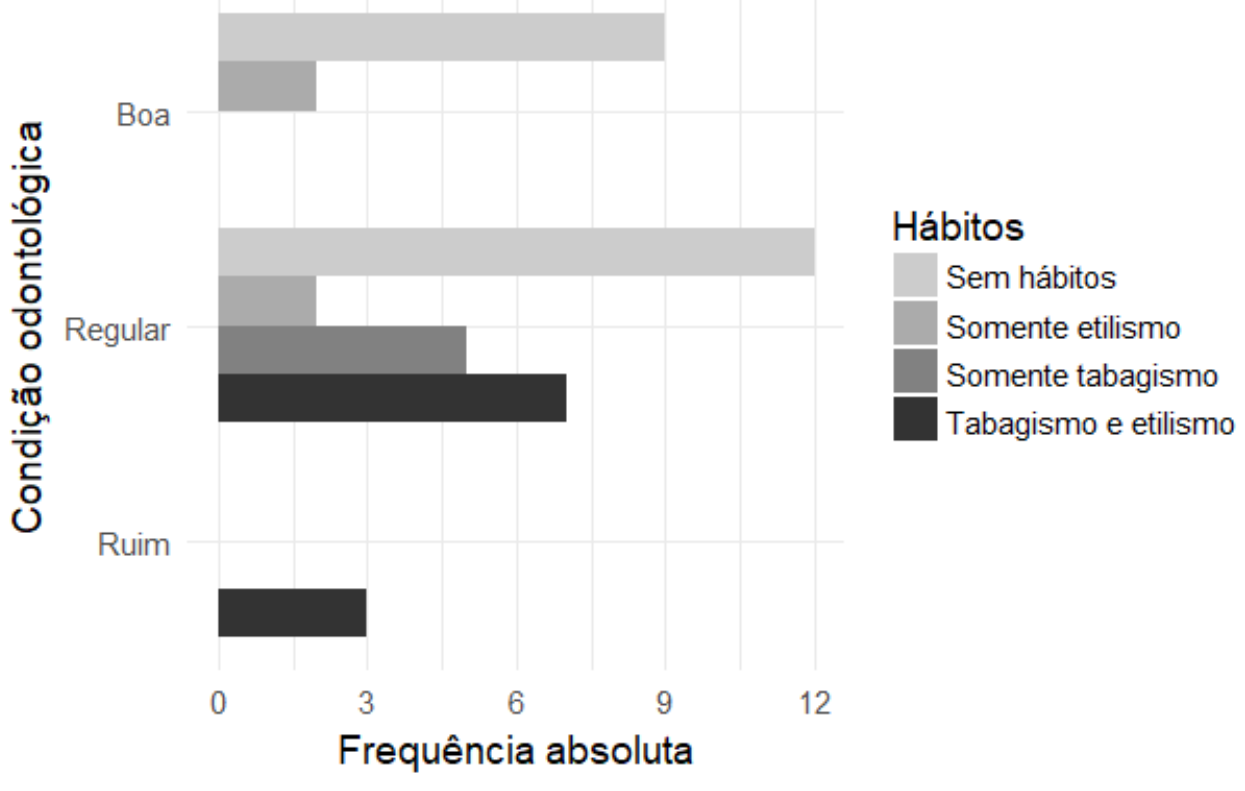

Fonte: Autores.

Observa-se na Tabela 1 que os focos orais são ausentes em 37,50\% dos pacientes, sendo 2,50\% dos indivíduos investigados apresentaram infecção por candidíase, $5 \%$ por abscessos dentoalveolares, e 27,50\% apresentaram raízes residuais presentes em cavidade bucal. E dentre as alterações bucais, as lesões traumáticas foram as mais frequentes, atingindo $42,50 \%$.

Tabela 1: Distribuição dos indivíduos em função da existência de focos infecciosos orais.

\begin{tabular}{ccc}
\hline VARIÁVEL & FREQUÊNCIA & $\%$ \\
\hline FOCOS ORAIS & 1 & $2,50 \%$ \\
Candidíase & 17 & $42,50 \%$ \\
Lesões traumáticas em cavidade bucal & 2 & $5,00 \%$ \\
Abscessos Dento Alveolares & 11 & $27,50 \%$ \\
Raízes residuais & 15 & $37,50 \%$ \\
\hline
\end{tabular}

Fonte: Autores.

Em relação ao índice de saburra lingual segundo a área, observa-se como detalhado na Tabela 2 que apenas 5,00\% e $17,50 \%$ dos pacientes não apresentaram saburra ou a mesma é classificada como subclínica, respectivamente, enquanto que 22,50\% apresentam até $1 / 3$ da língua, 32,50\% até 2/3 da língua e 22,50\% mais de 2/3 da língua. Já em relação ao índice de saburra lingual segundo a espessura, apenas $20 \%$ dos indivíduos possuíram saburra fina, ao passo que 47,50\% e 32,50\% apresentam-se com papilas linguais visíveis e sem visibilidade, respectivamente. 
Tabela 2: Distribuição dos indivíduos com relação ao Índice de Saburra Lingual quanto à área e espessura de Silness e Löe.

\begin{tabular}{ccc}
\hline VARIÁVEL & FREQUÊNCIA & $\%$ \\
\hline $\begin{array}{c}\text { INDICE DE SABURRA LINGUAL SEGUNDO A ÁREA } \\
\text { Sem saburra }\end{array}$ & 2 & $5,00 \%$ \\
Subclínica & 7 & $17,50 \%$ \\
Até 1/3 da língua & 9 & $22,50 \%$ \\
Até 2/3 da língua & 13 & $32,50 \%$ \\
Acima de 2/3 da língua & 9 & $22,50 \%$ \\
INDICE DE SABURRA LINGUAL SEGUNDO A ESPESSURA & 8 & $20,00 \%$ \\
Fina & 19 & $47,50 \%$ \\
Com papilas linguais visiveis & 13 & $32,50 \%$ \\
\hline
\end{tabular}

Fonte: Autores.

\section{Discussão}

A presença do biofilme oral pode influenciar no tratamento médico, devido aos fatores de virulência dos microorganismos que nela se encontram. Assim como, alterações bucais como a doença periodontal, cáries, necrose pulpar, lesões em mucosas, dentes fraturados ou infectados, traumas provocados por próteses fixas ou móveis podem também trazer para os pacientes repercussões negativas para suas condições sistêmicas (Silva, Bernardo, Avelar, Vasconcelos, \& Vasconcelos, 2017). As condições críticas que aumentam o risco de o paciente desenvolver doenças estão intimamente ligadas com a falta de um atendimento odontológico adequado na UTI, que tenha a função de controlar o biofilme, evitar e tratar a doença periodontal, e outras infecções bucais, bem como prevenir a infecção hospitalar (Sannapieco, 2002). Os resultados deste estudo revelaram que $27,50 \%$ e $65,00 \%$ dos pacientes apresentavam condição odontológica boa e regular, respectivamente. Tais dados reforçam os efeitos positivos da presença do cirurgião dentista dentro do ambiente de UTI, para manter sob vigilância e cuidados os tecidos e estruturas bucais.

Sabe-se que os cuidados bucais, quando realizados adequadamente, reduzem significativamente o aparecimento de pneumonia associada ao uso de ventilação artificial, nos pacientes em UTI (Cruz et al., 2014; Bezerra et al., 2020). Neste estudo visualizamos que, 7,50\% dos pacientes apresentaram condição odontológica ruim e que toda essa quantificação está diretamente relacionada com o uso do tubo oro traqueal (82,50\%). Porém esses dados não mantem associação direta com os $32,5 \%$ de pacientes que apresentavam diagnóstico de pneumonia como comorbidade. Em contrapartida, estudos mostram que após cinco dias de internação em UTI os pacientes que desenvolveram pneumonia nosocomial tinham sua etiologia bacteriana associada com a composição bacteriana do biofilme bucal (Azizi, \& Rezaee, 2012).

Além disso, foram avaliadas as condições odontológicas associadas aos hábitos deletérios à saúde bucal. O uso abusivo do drogas implica em efeitos nocivos tanto para a saúde sistêmica como da boca. Em relação à saúde bucal o alto consumo do álcool, por apresentar natureza agressiva da mucosa oral, está relacionado ao aumento do risco para desenvolver doenças gengivais e periodontais, bem como com o surgimento de lesões cariosas e dentre outras manifestações. Agregado a esses efeitos deletérios causados pelo consumo do álcool, os usuários normalmente apresentam negligência da higiene oral, favorecendo a formação do biofilme (Cabov et al., 2010). Já a nicotina age diretamente reduzindo a resposta imunológica do 
indivíduo, deixando-os mais vulneráveis a doenças sistêmicas e orais. Dentre as principais manifestações orais encontram- se as doenças periodontais, maior propensão ao surgimento de lesões cariosas e halitose (Cho, Hirsch, \& Johnstone, 2005). Com isso concluímos, com evidências amostrais do referido estudo, que a condição odontológica e os hábitos estejam significativamente associados, já que todos pacientes classificados com condição odontológica ruim possuíam ambos os hábitos de tabagismo e etilismo.

A avaliação da condição bucal e a necessidade de intervenção odontológica nos pacientes hospitalizados exige atuação direta de um cirurgião dentista habilitado em Odontologia Hospitalar. A Odontologia se faz necessária em âmbito crítico no diagnóstico e avaliação de alterações bucais, infecções, lesões traumáticas, cariosas e periodontais que representem risco ou desconforto para os pacientes hospitalizados (Marques et al., 2015).

Evidências científicas têm demonstrado a associação entre a saúde bucal e diversas doenças. E que, os microrganismos nativos bucais podem prejudicar à saúde podendo atuar como foco de disseminação de micro-organismos com efeitos patogênicos como: endocardite, doenças cardiovasculares, nascimento de neonatos, infecção de próteses articulares, controle metabólico do diabetes, doenças respiratórias, entre outros. A microbiota bucal e as toxinas provenientes do seu metabolismo podem comprometer a saúde sistêmica, por meio da disseminação via hematogênica ou por aspiração desse conteúdo intraoral (Amaral, Cortês, \& Pires, 2009).

Em 1911, o anatomista William Hunter publicou a teoria da infecção, afirmando que focos bucais causavam infecções a distância (Velzen, Abraham-Inpiji, \& Moorer, 1984). E que existe essa conexão por diversas vias de infecções orais e potenciais efeitos sistêmicos: toxinas e produtos infecciosos produzindo o efeito de bacteremia; injúrias metastáticas devido à presença de toxinas de bactérias orais na circulação sanguínea e o processo inflamatório que estes micro-organismos provocam (Hunter, 1911). Enfatizando mais uma vez a importância da atuação do cirurgião dentista no paciente crítico através da avaliação de focos infeciosos bucais, na qual observamos um número pouco expressivo de pacientes com infecções dento alveolares 5\% e fúngicas 2,5\%, por Candida albicans. Porém, foram encontradas 42,50\% de lesões traumáticas em cavidade bucal.

Estudiosos descrevem o biofilme bucal, a saburra lingual e o tubo orotraqueal como reservatórios para patógenos respiratórios. Oliveira, Carneiro, Fischer e Tinoco (2007) em sua amostra de 30 pacientes encontraram $70 \%$ dessas bactérias no biofilme dental, $63,33 \%$ no dorso da língua, $73,33 \%$ no tubo do respirador artificial e 43,33 \% em todas as áreas simultaneamente. O que torna importante os resultados encontrados nesta pesquisa, visto que $32,50 \%$ dos pacientes críticos avaliados apresentavam língua saburrosa até $2 / 3$ de extensão e de espessura de modo que as papilas linguais estivessem visíveis, $72,50 \%$ apresentavam algum biofilme dental e 82,50\% estavam sob ventilação mecânica, com tubo orotraqueal (Saldanha et al., 2015).

A cavidade oral não está isolada do corpo humano e consequentemente da saúde geral do indivíduo. Além do cirurgião dentista, todo profissional da saúde deve ter consciência da associação entre doença bucal e sistêmica, para poder prevenir, encaminhar e tratar os pacientes de maneira adequada (Kahn et al., 2008; Celik \& Eser, 2017). Mediante a importância de diagnosticar, controlar e prevenir doenças de pacientes em UTIs é de grande necessidade a participação de um cirurgião-dentista na estimulação de ações preventivas e clínicas direcionadas à promoção de saúde bucal em pacientes críticos (Roberts, \& Moule, 2011; Miranda, 2016; Londe, Ferreira, Novaes, Barbosa, \& Miranda, 2017).

Diante destes dados fica elucidado que com o atendimento odontológico dos pacientes hospitalizados e a eliminação dos fatores de risco bucais ocorre a redução do aparecimento de possíveis infecções hospitalares e/ou agravamento à saúde dos pacientes hospitalizados. Ainda, pesquisas são necessárias para determinar o impacto da saúde oral frente à saúde sistêmica de pacientes internados em UTIs e que estes atendimentos devem ser preconizados. 


\section{Conclusão}

Em pacientes internados em UTI, observa-se uma higiene bucal deficiente, tornando-se o biofilme um reservatório propício de micro-organismos que podem agravar em um processo infeccioso localizado, ocasionando problemas sistêmicos, como a pneumonia nosocomial. Assim sendo, conclui-se que é de extrema importância a participação de um cirurgião-dentista na equipe interdisciplinar de uma UTI para avaliar, diagnosticar, tratar e acompanhar o acometimento da saúde bucal dos referidos pacientes. Portanto, é necessário que novas pesquisas sejam desenvolvidas reforçando a importância da necessidade da implantação da Odontologia Hospitalar para pacientes internados em UTI.

\section{Referências}

Amaral, S. M., Cortês, A. Q., \& Pires, F. R. (2009). Pneumonia nosocomial: importância do microambiente oral. J Bras Pneumol, 35 (11), $1116-1124$.

Araújo, L. J. S. (2015). Cuidados Periodontais nas Unidades de Terapia Intensiva com Pacientes Idosos. Anais CIEH, 2(1), ISSN 2318-0854.

Azizi, A., \& Rezaee, M. (2012). Comparison of periodontal status in gingival oral lichen planus patients and healthy subjects. Dermatol Res Pract, (25), 561622 .

Baeder, F. M., Cabral, G., Prokopowitch, M. P., Arak, I., Duarte, A. T. I., \& Santos, D. A. (2012). Condição Odontológica em Pacientes Internados em Unidade de Terapia Intensiva. Pesq Bras Odontoped Clin Integr, 12(4), 517-520.

Bezerra, T.P., Campêlo, C.S.P., Oliveira, A.F., Forte, C.P.F., Gomes, A.K.P., Silva, P.G.B., et al. (2020). Pneumonia and the role of dentistry on ICU Staff: 8year analysis of health indicators. Brazilian Journal of Oral Sciences, 19, 1-8.

Cabov, T., Macan, D., Husedzinović, I., Skrlin-Subić, J., Bosnjak, D., Sestan-Crnek S., et al. (2010). The impact of oral health and 0.2\% chlorhexidine oral gel on the prevalence of nosocomial infections in surgical intensive-care patients: a randomized placebo-controlled study. Wien Klin Wochenschr, 122(13-14), 397-404.

Celik, G.G., \& Eser I. (2017). Examination of intensive care unit patients' oral health. International Journal of Nursing Practice, 1-9.

Cho, C. M., Hirsch, R., \& Johnstone, S. (2005). General and oral health implications of cannabis use. Australian Dental Journal, $50(2), 70-74$.

Cruz, M. K., Morais, T. M. N., \& Trevisani, D. M. (2014). Avaliação clínica da cavidade bucal de pacientes internados em unidade de terapia intensiva de um hospital de emergência. Rev Bras Ter Intensiva, 26(4), 379-383.

Estrela, C. (Org.). (2018). Metodologia científica: ciência, ensino, pesquisa. (Scientific methodology: science, teaching research) (3rd Edition). Porto Alegre: Artes Médicas.

Ferraz, E. G., Silva, L. R., Sarmento, V. A., Campos, E. J., Oliveira, T. F. L. O., \& Magalhães, J. C. (2014). Avaliação dos hábitos de higiene bucal em pacientes pediátricos obesos. Pediatria Moderna, 14(50), 69-74.

Gaetti-Jardim, E. C., Setti, J. S., Cheade, M. F. M., \& Mendonça, J. C. G. (2013). Atenção odontológica a pacientes hospitalizados: revisão da literatura e proposta de protocolo de higiene oral. Rev Bras Ciência Saúde, 11.

Hunter, W. (1911). The role of sepsis and antisepsis in medicine. Lancet, 11, 79-86.

Kahn, S., Garcia, K. H., Júnior, J. G., Namen, F. M., Machado, W. A. S., Júnior, J. A. S., Sardenberg, E. M. S., \& Egreja, A. M. (2008). Avaliação da existência de controle de infecção oral nos pacientes internados em hospitais do estado do Rio de Janeiro. Ciência \& Saúde Coletiva, 13(6), 1825-1831.

Londe, L. P., Ferreira, J. A., Novaes, L. A. C. F., Barbosa, R. S., \& Miranda, A. F. (2017). Pneumonia nosocomial e sua relação com a saúde bucal. Revista Ciências e Odontologia, 1(1), 24-28.

Marques, L. A. R. V., Lotif, M. A. L., Neto, E. M. R., Dantas, T. S., Soares, J. L., Melo, J. A., \& Lobo, P. L. D. (2015). Abuso de drogas e suas consequências na saúde oral: uma revisão de literatura. Arquivo Brasileiro de Odontologia, 11(1).

Miranda, A. F. (2016). Oral Health and Care at Intensive Care Units. Journal of Nursing \& Care, 5(1), 1-6.

Morais, T. M. N., Silva, A. S., Avis, A. L. R. O., Souza, P. H. R., Knobel, E., \& Camargo, L. F. A. C. (2006). Importância da Atuação Odontológica em Pacientes Internados em Unidade de Terapia Intensiva. Revista Brasileira de Terapia Intensiva, 18(4).

Oliveira, L. C. B. S., Carneiro, P. P. M., Fischer, R. G., \& Tinoco, B. E. M. (2007). A presença de patógenos respiratórios no biofilme bucal de pacientes com pneumonia nosocomial. Rev Bras Ter Intensiva, 19(4), 428-433.

Oliveira, T. F. L., Filho, I. S. G., Passos, J. S., Cruz, S. S., Oliveira, M. T., Trindade, S. C., Machado, A. S., Coelho, J. M. F., Santos, C. M. L., \& Cerqueira, E. M. M. (2011). Fatores Associados à Pneumonia Nosocomial em Indivíduos Hospitalizados. Rev. Assoc. Med. Bras., 57(6).

Pereira, A. S., Shitsuka, D. M., Parreira, F. J., \& Shitsuka, R. (2018). Scientific research methodology (1rd Edition). Santa Maria, RS: UFSM, NTE. 
Research, Society and Development, v. 10, n. 6, e6910615365, 2021

(CC BY 4.0) | ISSN 2525-3409 | DOI: http://dx.doi.org/10.33448/rsd-v10i6.15365

Roberts, N., \& Moule P. (2015). Chlorhexidine and tooth-brushing as prevention strategies in reducing ventilator-associated pneumonia rates. Nurs Crit Care, Bristol, 16(6), 295-302.

Saldanha, K. F. D., Costa, D. C., Pinto, S. F., \& Gaetti-Jardim, E. C. (2015). Avaliação do índice de higiene oral do paciente crítico. Arch Health Invest, 4(6), 47-53, ISSN 2317-300.

Sannapieco, F. A. (2002). Relação entre Doença Periodontal e Doenças Respiratórias. Medicina Periodontal, 83-97.

São Paulo. (2012). Secretaria de Saúde. Manual de odontologia hospitalar. São Paulo: Grupo Técnico de Odontologia Hospitalar.

Schlesener, V. R. F., Rosa, U. D., \& Raupp, S. M. M. (2012). O Cuidado com a Saúde Bucal de Pacientes em UTI. Cinergis, 13(1), $73-77$.

Silva, I. V. S., Bernardo, N. M. X., Avelar, W. V., Vasconcelos, M. G., \& Vasconcelos, R. G. (2017). A importância dos odontólogos na rede hospitalar: um enfoque sobre a unidade de terapia intensiva. Odontol. Clin.-Cient., 16(2), 79-84.

Velzen, S. K., Abraham-Inpiji, L., \& Moorer, W. R. (1984). Plaque and systemic disease: a reappraisal of the focal infection concept. J. Clin. Periodont., 11, 209-220. 